$63^{\text {ème }}$ Congrès de la SFCO, 02001 (2015)

DOI:10.1051/sfco/20156302001

(C) Owned by the authors, published by EDP Sciences, 2015

\title{
COMMUNICATION
}

\section{Planification simplifiée en chirurgie orthognathique bi-maxillaire}

\author{
Afota $\mathrm{F}^{* * * *}$, Busson $\mathrm{F}^{* *}$, Manière $\mathrm{A}^{* *}$, Tabet $\mathrm{C}^{*}$, Savoldelli $\mathrm{C}^{* * * *}$ \\ * Service Chirurgie Orale et Maxillo-faciale, Institut Universitaire de la Face et du Cou, CHU NICE, 31 avenue \\ Valombrose, 06000 Nice \\ ** Service Odontologie, Hopital St Roch, CHU Nice, 5 rue Pierre Devoluy, 06000 Nice
}

Le résultat occlusal et esthétique en chirurgie orthognathique dépend du bilan pré-opératoire mais également de la planification chirurgicale. Cette planification ou « set up » chirurgical va dans le sens d'une anticipation du futur plan d'occlusion. La réalisation de gouttière chirurgicale sur articulateur est chronophage et parfois source d'erreurs. Nous présentons notre expérience d'une planification simplifiée des patients opérés d'ostéotomie bi-maxillaire dans le service de Chirurgie Orale et Maxillo-faciale du CHU de Nice. Chaque planification était réalisée 3 jours avant la chirurgie. Le transfert des relations inter maxillaires (RIM) sur articulateur s'effectuait classiquement en y ajoutant un système de mobilisation des modèles appelés P3D. Les mouvements des modèles facilités par ce système $\mathrm{P} 3 \mathrm{D}$ étaient réalisés après une analyse clinique esthétique et occlusal et un set-up céphalométrique.

De notre expérience, le gain de temps était significatif avec une bonne fiabilité de résultat.

Nom et adresse du conférencier

Franck AFOTA

Service Chirurgie Orale et Maxillo-faciale

Institut Universitaire de la Face et du Cou, CHU NICE

31 avenue Valombrose

06000 Nice (France)

franck.afota@hotmail.fr

This is an Open Access article distributed under the terms of the Creative Commons Attribution License 4.0, which permits unrestricted use, distribution, and reproduction in any medium, provided the original work is properly cited. 